\title{
CRISPR-mediated modification of DNA methylation pattern in the new era of cancer therapy
}

\author{
Faezeh Maroufi ${ }^{1}$ (D) Amirhosein Maali ${ }^{2,3}$ (iD), Meghdad Abdollahpour-Alitappeh ${ }^{4}$ (D), \\ Mohammad Hossein Ahmadi ${ }^{1}$ (D) \& Mehdi Azad*,1 (D) \\ 'Department of Medical Laboratory Sciences, Faculty of Allied Medicine, Qazvin University of Medical Sciences, Qazvin, Iran \\ ${ }^{2}$ Student Research Committee, Pasteur institute of Iran, Tehran, Iran \\ ${ }^{3}$ Department of Medical Biotechnology, Faculty of Allied Medicine, Qazvin University of Medical Sciences, Qazvin, Iran \\ ${ }^{4}$ Cellular \& Molecular Biology Research Center, Larestan University of Medical Sciences, Larestan, Iran \\ *Author for correspondence: Tel..: +982 833359 501; haematologicca@gmail.com
}

In the last 2 decades, a wide variety of studies have been conducted on epigenetics and its role in various cancers. A major mechanism of epigenetic regulation is DNA methylation, including aberrant DNA methylation variations such as hypermethylation and hypomethylation in the promoters of critical genes, which are commonly detected in tumors and mark the early stages of cancer development. Therefore, epigenetic therapy has been of special importance in the last decade for cancer treatment. In epigenetic therapy, all efforts are made to modulate gene expression to the normal status. Importantly, recent studies have shown that epigenetic therapy is focusing on the new gene editing technology, CRISPR-Cas9. This tool was found to be able to effectively modulate gene expression and alter almost any sequence in the genome of cells, resulting in events such as a change in acetylation, methylation, or histone modifications. Of note, the CRISPR-Cas9 system can be used for the treatment of cancers caused by epigenetic alterations. The CRISPR-Cas9 system has greater advantages than other available methods, including potent activity, easy design and high velocity as well as the ability to target any DNA or RNA site. In this review, we described epigenetic modulators, which can be used in the CRISPR-Cas9 system, as well as their functions in gene expression alterations that lead to cancer initiation and progression. In addition, we surveyed various species of CRISPR-dead Cas9 (dCas9) systems, a mutant version of Cas9 with no endonuclease activity. Such systems are applicable in epigenetic therapy for gene expression modulation through chemical group editing on nucleosomes and chromatin remodeling, which finally return the cell to the normal status and prevent cancer progression.

First draft submitted: 18 March 2020; Accepted for publication: 11 September 2020; Published online: 13 November 2020

Keywords: cancer therapy • CRISPR-Cas9 • DNA methylation • epigenetics • gene editing

Cancer is one of the most common diseases around the world, causing a huge psychological and economic burden for society and many deaths worldwide [1]. Cancers are developed with both genetic and epigenetic abnormalities. More importantly, epigenetic abnormalities are even more frequent in most human cancers. Heritable modifications in gene expression caused by epigenetic factors can lead to suppression or activation of particular genes without directly altering DNA sequences [2]. Epigenetic profiles change in different steps of tumor development and progression. DNA methylation, as one of the major mechanisms of epigenetic regulation, not only can control gene expression but also plays a well-established role in the pathogenesis of many cancers [3]. DNA methylation occurs primarily on cytosine residues near the gene promoters with a higher concentration of $\mathrm{CpG}$ sites, known as $\mathrm{CpG}$ islands, via DNA methyltransferase (DNMT). Subsequently, chromatins are remodeled and gene transcription is inhibited through complexes such as histone deacetylase (HDAC), and some proteins such as heterochromatin protein 1 . However, such alterations are reversible and return to the normal state through other enzymes such as ten-eleven translocation methylcytosine dioxygenase (TET).

Future Medicine 